\title{
Comparative Analysis of Thermoelectric Generators Parameters
}

\author{
M. BensaAda, F. Metehri, S. Della Krachai \\ Centre of Satellite Development, Power System Laboratory, BP 4065 Ibn Rochd USTO, Oran, ALGERIA, 31130 \\ Tel: + 21341560329 - Fax: + 21341560301
}

\begin{abstract}
Other sources of energy in space applications remain unexploited such as heat. Indeed the exchange of heat is considered generally on board spacecraft as hostile, destructive and undesirable, thereby a different means are used to reduce its effect on board spacecraft. Heat being an important source of energy, it remains badly exploited on spacecraft and its applications remain limited. We present in this paper one of the methods used to convert heat energy to electrical energy by using thermoelectric device, the goal becomes therefore to choose a device capable to give a best performances through a comparative analysis between different commercial thermoelectric generator devices to be able subsequently to make a choice of the component to be used for future design. This analysis will allow us thereafter to design a thermoelectric generator as secondary power source for small satellite by exploiting the external thermal properties of the spacecraft on orbit.
\end{abstract}

Keywords-Low Earth Orbit, photo-generation, spacecraft, thermoelectric generator, heat

Received: October 16, 2020. Revised: December 26, 2020. Accepted: January 19, 2021. Published: January 30, 2021.

\section{Introduction}

The thermoelectric module (TEM) devices can typically be classified into thermoelectric coolers (TEC) and thermoelectric generators (TEG). TEC convert electrical energy into a temperature gradient (Peltier effect), whereas TEG is a device for directly converting thermal energy into electrical energy based on the Seebeck effect and it has presented urgent potential in the case of waste heat recovery. The TEG have many advantages such as no moving mechanical parts, long-lived, quiet, environmentally friendly and requiring little maintenance [1]. Being basically solidstate energy converters with no mechanical and moving parts, TEM devices can provide the benefits of noiselessness, compactness, reliability, and stability. However, having poor energy conversion efficiency, both TEC and TEG were largely considered for niche applications due to their unique advantages. In general, TEC are capable of cooling and refrigeration, such as in specialized coolers for optoelectronic and electronic components and portable electric food coolers/heaters. They are especially useful for precise temperature control applications where accuracy, simplicity, reliability, and compactness are dominant concerns. On the other hand, TEG can be used to harness waste heat with available temperature difference and produce electric power, such as for power generation in spacecraft systems and as thermocouples for temperature measurement. With the rising costs of fossil fuels and the innovative technologies of wasteheat recovery, TEG devices are becoming more prominent, especially in applications [2].

Temperature distribution on a low earth orbit spacecraft surface is crucial for determination of the devices and electronics lifetime and proper operations, which will be carried by the satellite. These devices include batteries and electronic circuits. The proper operation of these components is sensitive to the values and changes of surrounding temperature. So this paper consists in the first time to make a comparative study between two different types of TEG in order to be able to subsequently choose the TEG with the best performances by analyse and experimental characterization of the electrical behaviour of the thermoelectric generators. The experimental setup can be used for any type of TEG, for this paper; SP1848 and TEC1-12705 are used to complete the work.

\section{Principal of Thermoelectric Generator}

Thermoelectric generators are all solid-state devices that convert heat into electricity [3]. Thermoelectric systems can be easily designed to operate with small heat sources and small temperature differences. Such small generators could be mass produced for use in automotive waste heat recovery or home co-generation of heat and electricity. Thermoelectric have even been miniaturized to harvest body heat for powering a wristwatch. Phenomenon in which a voltage develops when two different conductors are joined together and the junction is heated [4]. The thermal EMF $\Delta V$ is given by:

$$
\Delta V=\left(\alpha_{A}-\alpha_{B}\right) \cdot \Delta T=\alpha_{A B} \cdot \Delta T
$$

The quantity $\alpha_{A B}$ is called the relative Seebeck coefficient and is the difference between absolute Seebeck coefficients of the two materials $\mathrm{A}$ and $\mathrm{B}$.

\subsection{System Modeling}

The construction of both TECs and TEGs is basically the same, and consequently the same equivalent circuit can be used to simulate their behavior. The basic unit of a TEM is a thermocouple that consists of a p-type and n-type pellet couple interconnected by metal. A thermoelectric produces electrical power from heat flow across a temperature gradient [5]. As the heat flows from hot to cold, free charge carriers (electrons or holes) in the material are also driven to the cold end as shown in Fig. 1. 


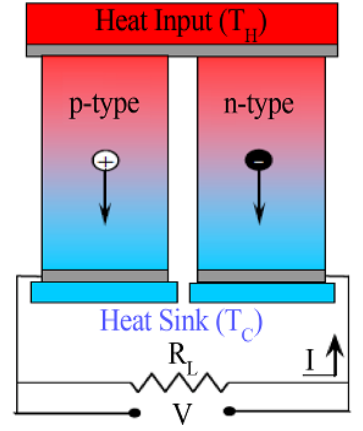

(a)

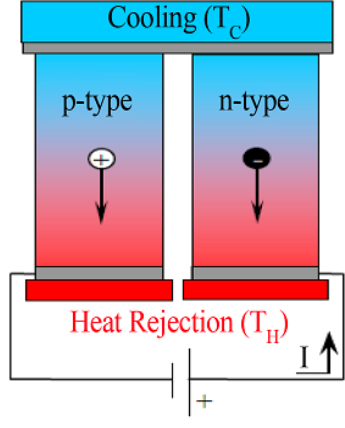

(b)
Figure 1. Thermoelectric (a) power generation by Seebeck effect, (b) cooling by Peltier effect.

To Model the TEG system there are two commercially available thermoelectric generators are experimentally studied for the proposed system, SP1848 and TEC1-12705. In Fig. 1 a TEG consists of two dissimilar materials, n-type and p-type semiconductors, connected thermally in parallel and electrically in series.

The voltage produced is proportional to the temperature difference between the two junctions. This is given by:

$$
V=\alpha\left(T_{H}-T_{C}\right)
$$

$T_{H}$ and $T_{C}$ are respectively the two junctions with $T_{H}$ less than $T_{C}$.

\subsection{Significant Parameters}

The most important parameters in a thermoelectric material are the Seebeck coefficient and figure of merit [7]. The Seebeck coefficient is a measure of the transported entropy per charge carrier. The figure of merit $(Z T)$ is expressed as:

$$
Z T=\frac{\alpha^{2} \sigma}{k} T
$$

The figure of merit varies directly with the Seebeck coefficient and electrical conductivity $(\sigma)$ and inversely with thermal conductivity $(k)$. An increase in electrical conductivity reduces the losses caused due to Joule heating. A decrease in the thermal conductivity would limit the amount of heat passing through the module without being converted into power [8].

\section{Derivation TEG Construction and Configuration}

A typical thermoelectric module is made of multiple leg pairs, where each leg pair is made of two semiconductor materials made from $\mathrm{p}$ and $\mathrm{n}$ type semiconductor materials [6]. The combination of these multiple leg pairs is held together by a top and bottom plate, typically made of a ceramic-like aluminium nitride. This ensures electrical insulation and structural support. The leg pairs are connected in such a way that they are in series electrically and in parallel thermally. The pellets, tabs, and ceramic plates form a layered configuration, as seen in Fig. 2.

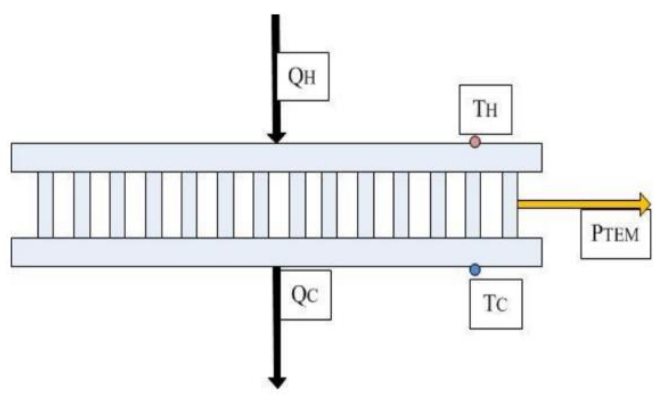

Figure 2. TEG in power generation configuration.

The power generation arrangement where heat enters the hot side of the module and is rejected from the cold side of the module. The most commonly used approach for calculating power generation is a one-dimensional approach. Using the model developed by Angrist [9-10], the heat flow into the hot side of the module, $Q_{H}$ and the heat flow out of the module $Q_{C}$ are expressed as:

$$
\begin{gathered}
Q_{H}=I \alpha_{p, n} T_{H}+k\left(T_{H}-T_{C}\right)-I^{2} \frac{R}{2} \\
Q_{H}=I \alpha_{p, n} T_{C}+k\left(T_{H}-T_{C}\right)-I^{2} \frac{R}{2}
\end{gathered}
$$

where $I$ is the current flowing through the module and is the overall Seebeck coefficient, which is the product of the difference of the individual Seebeck coefficients of the two constituent materials and the number of leg pairs. $T_{H}$ and $T_{C}$ are the temperatures on the hot and cold sides of the module. $\mathrm{R}$ is the electrical resistance, which includes the leg and metal connector contact resistance. The thermal conductance for the module can be expressed as the ratio of the actual heat rate delivered to the module to the temperature difference across the module when the module is in the open-circuit state, expressed as:

$$
k=\frac{Q_{H}}{T_{H}-T_{C}}
$$

These expressions are developed by solving the heat diffusion equation in the device and applying a fixed temperature and the Peltier effect model at the surfaces. From energy conservation, the electrical power output of a thermoelectric module is expressed as:

$$
\begin{gathered}
P_{T E G}=\left(Q_{H}-Q_{C}\right) \\
P_{T E G}=I \alpha_{p, n}\left(T_{H}-T_{C}\right)-I^{2} R
\end{gathered}
$$

The efficiency of the module can be expressed as the ratio of the power output to the heat input, and is given as:

$$
\begin{gathered}
\eta=\frac{P_{T E G}}{Q_{H}} \\
\eta=\frac{I \alpha_{p, n}\left(T_{H}-T_{C}\right)-I^{2} R}{I \alpha_{p, n} T_{H}+k\left(T_{H}-T_{C}\right)-I^{2} \frac{R}{2}}
\end{gathered}
$$

Most of the modules commercially available today can be classified as low temperature Research conducted on low 
temperature waste heat recovery systems have demonstrated the potential viability of thermoelectric systems.

\section{Test Results}

For the electrical characterisation of TEGs a test bed was developed as shown in Fig. 3. Inside the bed test tows type of thermoelectric generator was tested: SP1848 and TEC112705 .

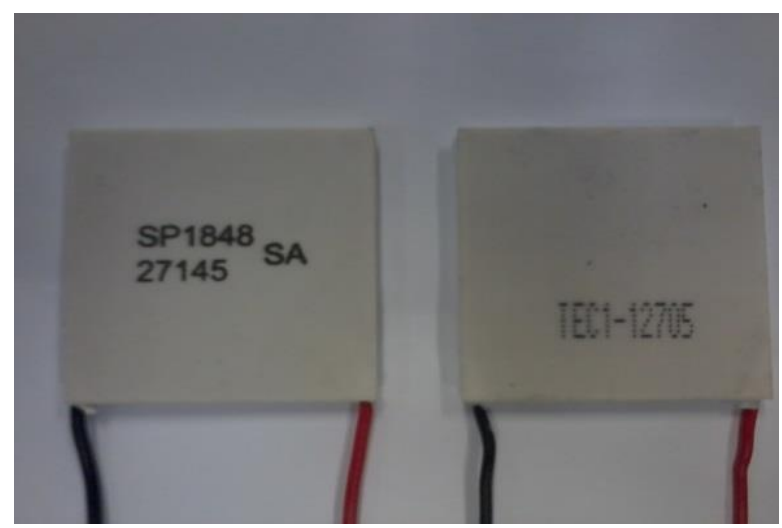

Figure 3. Thermoelectric devices of the test bed

Before the system performance testing, a performance analysis and evaluation should be achieved by means of testing an individual TEG. Considering the limited working temperature, less than $20^{\circ} \mathrm{C}$, the maximum working temperature is set to $100^{\circ} \mathrm{C}$. Different clamp loads are chosen and applied to the TEG for better electrical performance. The accurate electrical characteristics could be obtained by sweeping the electrical loads at different values and maintaining the temperature difference at the same time. The test bed consists of a power supply, a shunt resistor, an aluminium heat sink, and a couple of thermocouples to measure the temperatures. The data from the thermocouples as well as the current and voltage are recorded. The difference temperature of the two junctions depend strongly on the heat source as well on the heat sink combined with the air cooler or any other method used. The block diagram of the setup is depicted in Fig. 4.

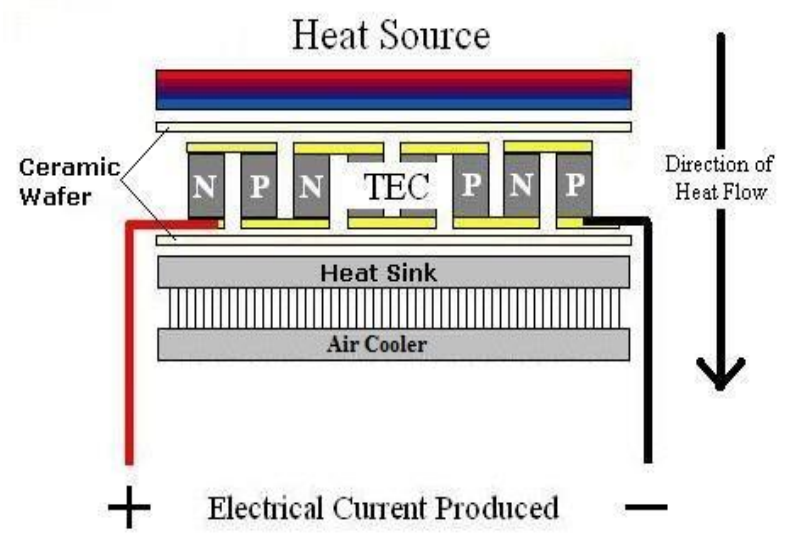

Figure 4. Block diagram of the test bed.

The experimental testbed consists of to measure the temperature of both sides temperature of the TEG $T_{H}$ and $T_{C}$. The voltage and current are as well measured for different configurations; open circuit, short circuit and for different values of load. As a source of heat, a hot air gun was used with the possibility to vary a temperature. To increase the difference temperature $\left(T_{H}-T_{C}\right)$ represented in the figures below by $(\Delta T)$ a fan is used on a cold side.
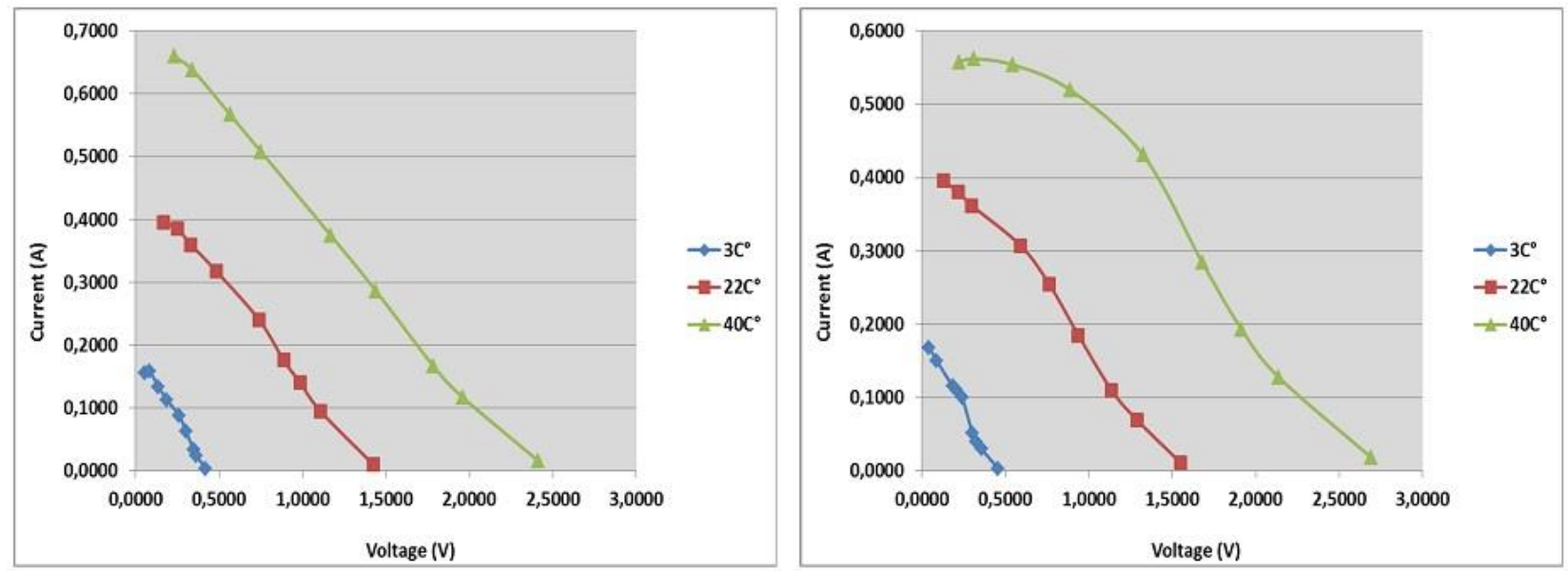

Figure 5. TEC1-12705 (I-V) Characteristic in the left side and SP1848 (I-V) Characteristic in the right side. 

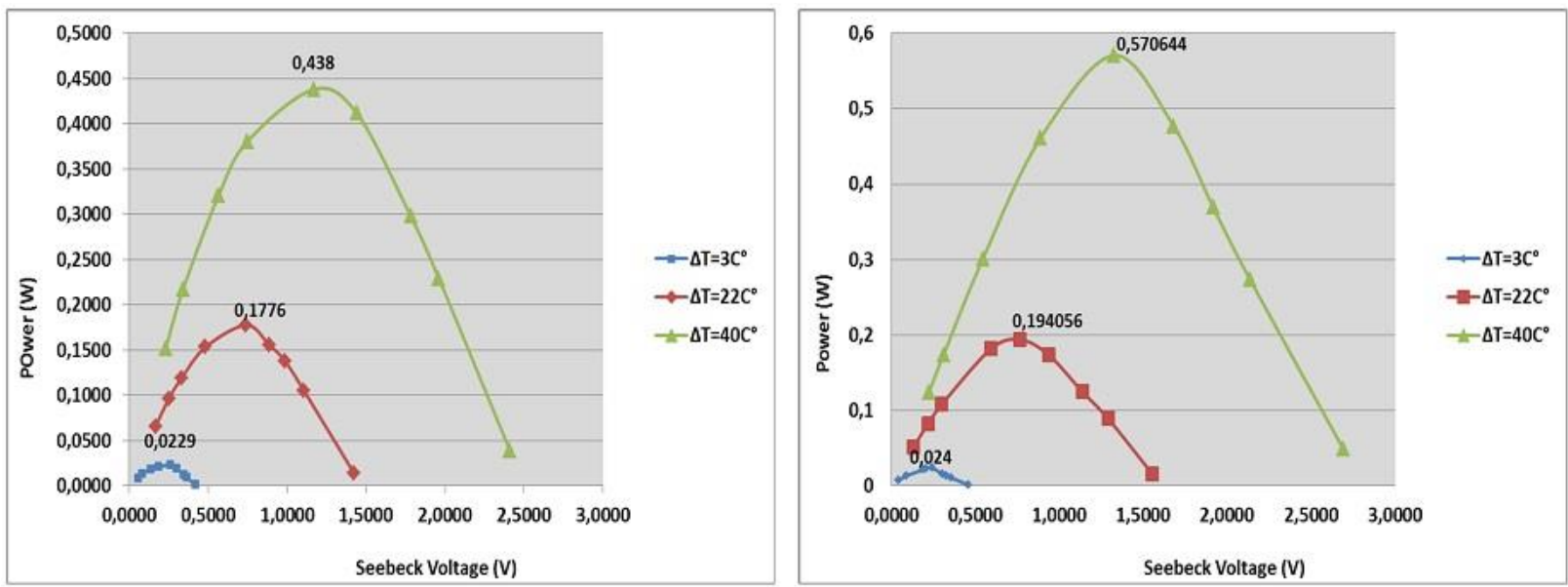

Figure 6. TEC1-12705 P-V Characteristic in the left side and TEG SP1848 (P-V) Characteristic in the right side.

The voltage/current characteristics of a generator include different aspects like the open circuit voltage $\left(V_{O C}\right)$ and short circuit current $\left(I_{S C}\right)$ represented by the Fig. 5, the generated power and the internal resistance. The junction temperature-dependent behaviour of a TEG for different values of the load resistance, the power generated by the SP1848 is slightly better than the TEC1-12705. Fig. 6 for the both TEGs show the P-V characteristic, the power delivered by the SP1848 offer a better efficiency than the TEC112705 , the power difference is around $133 \mathrm{mw}$. To maximize the power output a maximum power point (MPP) can be envisaged. Of course, In particular, for transient thermal conditions, an MPP could be interesting.

\section{Conclusion}

Inside the structure of satellites heat flows occur that are driven by environmental (sun radiation, etc.) and operational loads (electronics, etc.). These heat flows are unused so far, although there is a potential for generating electrical power in general. Thereby the generated electrical power can be used for an increase of the satellites efficiency by a feed-in to the main electrical power system. Through this work we focused on description and analyze the performance of particular commercial TEG devices. In general it is feasible to use low power thermoelectric generators that are manufactured for terrestrial applications in space. Because of the low efficiency of available TEG technology this is not sufficient today, on the other hand if we foreseen to use TEG on spacecraft, the thermoelectric generator pack should be designed so that the junction temperature must be the maximum possible in order to have a better efficiency. In general the TEG SP1848 present a better performance than TEC1-12705 thereby the thermoelectric pack will be designed with (SP1848) and will be the subject of future work.

\section{ACKNOWLEDGEMENTS}

This research is co-supported by department of Space Instrumentations, Algerian Space Agency / Centre of Satellites Development

\section{REFERENCES}

[1] A.F. Ioffe, Semiconductor Thermoelements and Thermoelectric Cooling (London, UK: Infoserch Limited, 1957).

[2] R. Ahiska, S. Dislitas, Computer controlled test system for measuring the parameters of the real thermoelectric module, Energy Convers. Manag. 52 (2011) 27-36.
[3] Müller E., Stiewe C., Rowe D., and Williams S., 2006, "Precision Thermal Conductivity Measurements of materials," Thermoelectrics Handbook Macro to Nano, pp. 26-8 - 26-9.

[4] Rowe D. M., 2006, Thermoelectric Handbook: Macro to nano, CRC/Taylor \& Francis.

[5] P. Dziurdzia. 2010. Modeling and Simulation of Thermoelectric Energy Harvesting Processes.

[6] S. Lineykin and S. Ben-Yaakov. 2007. Modeling and Analysis of Thermoelectric Modules. IEEE Trans. Ind. Appl. 43(2): 505-512.

[7] Muto A., 2008, "Device Testing and Characterization of Thermoelectric Nano Composites," MS Thesis, Mechanical Engineering, Massachusetts Institute of Technology.

[8] G. Snyder. 2008. Small Thermoelectric Generators. The Electromechanical Society Interface. 2: 54-56. K.T. Zorbas, E. Hatzikraniotis, and K.M. Praskevopoulos, "Power and Efficiency Calculation in Commercial TEG and Application in Wasted Heat Recovery in Automobile" 5th European Conference on Thermoelectrics, paper 30, Odessa, September 2007.

[9] Platzek, G. Bastian, K.-H. Förderer, D. Tatarinov, A. Vogelsang, H. Hupe, H. Platzek, M. Niecknig, and M. Preller, "A Thermoelectric Power Generator for the Metalworking Industry,"28th International Conference on Thermoelectrics, Freiburg, July 2009.

[10] L. Li, Z. Chen, M. Zhou, R. Huang, "Developments in semiconductor thermoelectric materials, “ Front. Energy, 2011, 5(2), pp. 125-136.

\section{Creative Commons Attribution License 4.0 (Attribution 4.0 International, CC BY 4.0)}

This article is published under the terms of the Creative Commons Attribution License 4.0 https://creativecommons.org/licenses/by/4.0/deed.en_US 